Mathematical Sciences and Applications

E-NOTES

MATHEMATICAL

SCIENCES

AND APPLICATIONS

E-NOTES

https://doi.org/110.36753 /mathenot.799596

10 (1) 45-49 (2022) - Research Article

ISSN: 2147-6268

(c) MSAEN

\title{
An Analytical Approach to an Elastic Circular Rod Equation
}

\author{
Zehra Pinar
}

\begin{abstract}
The size-dependent longitudinal and torsional dynamic problems for small-scaled rods have importance in two-phase media. The special case of the elastic rod equation such as magneto-electro circular equation are seen in the literature commonly, but in this work, the generalized form of the nonlinear elastic circular equation, which was not studied in the literature, is considered. The exact solutions are obtained via Mathieu approximation method with a novel proposed ansatz. Obtained solutions are discussed and illustrated in details. We believe that the proposed results will be key part of further analytical and numerical studies for waves in the dispersive medium with reaction.
\end{abstract}

Keywords: Mathieu approximation method; the elastic rod equation; travelling wave solutions.

AMS Subject Classification (2020): Primary: 35CXX ; Secondary: 35BXX; 35QXX.

\section{Introduction}

Modeling a wide range of problems related to different research areas such as fluid mechanics, plasma physics, optical fibers, biology, solid state physics, chemical kinematics, chemical physics and chemistry, is done by using partial differential equations (PDEs). Therefore, PDEs are of paramount importance among researchers. In particular, nonlinear wave propagation, diffusion, reaction and convection are very important. Thus, the longitudinal wave equation (LWE) arising in a magneto-electro-elastic circular rod is a major problem that rods have been used for transmission and owing to their superior electrical, mechanical, optical and other physical and chemical properties, areas of use vary [18]. Firstly, the models of LWE can be examined. The most known model for long finite-amplitude waves is the Korteweg-de Vries (KdV) equation but the dispersive term is ignored, so the non-linearity is dominant. Then, there were many attempts to improve the model via using extra nonlinear terms involving second-order and a third-order derivative. Hence, the aim is focused on finite-length and finite-amplitude waves. Respect to material of the rod, the equation is reduced to Benjamin-Bona-Mahony (BBM) equation which is known as an alternative to the $\mathrm{KdV}$ equation for modelling long finite-amplitude waves. Although, no matter how thin the rod is, it is always assumed three-dimensional, when the rod diameter is much smaller than the axial length scale, it is reasonable to expect that approximate one-dimensional equations (rod equations) can give a good description of the motion of the rod. Many modifications of rod equations are seen in the literature and these equations ignore many properties 
of rod and constraining terms $[8,12,13,17,18,19]$. In this work, the considered rod equation was not studied before in the literature and it contains physical and chemical properties of the rod. The generalized form of the elastic rod equation $[2,3,7]$ is determined

$$
u_{t t}-c_{0}^{2}\left(1+n a_{n}\left(u_{x}\right)^{2}\right)_{x x}-\frac{\nu^{2} J_{\rho}}{s} u_{t t x x}=F(t),
$$

where $F(t)$ is the forcing term, $s$ is the cross-section area of the rod, $J_{\rho}$ is the polar moment of inertia, $c_{0}^{2}\left(=\frac{E}{\rho}\right)$ is the square of the linear elastic longitudinal wave velocity, $E$ is the modulus of elasticity (Young's modulus), $\rho$ is the density of the rod, is the Poisson ratio, $n$ is an integer, $a_{n}$ is material constants of the rod.

In case of the soft-nonlinear materials $a_{n}$ is less than zero, whereas for hard-nonlinear materials such as rubbers polymers and some metals $a_{n}$ is greater than zero. As it is seen that, Eq.(1.1) is also material depended equation (model). As can be seen, when $F(t)=0$, Eq.(1.1) is reduced to the well-known rod equation and also when $a_{n}=0$ under adequate assumptions can be converted to classical wave equation for an elastic thin rod [7]. In the literature, solitary wave solutions of the Eq.(1.1) are obtained for $n=2$ and $n=3$ by simplicity [17, 19, 20]. Generally, the ansatz is determined after reducing and integrating the Eq.(1.1) [1, 6, 7]. But the degree of the ansatz is fractional that is not valid. In this work, the Eq.(1.1) is considered in the original form and the degree of the ansatz is determined in a novel way. Till now, the degree of ansatz is determined by balancing principle which is generally given for the power-law non-linearity. The reason of the transformations and integration is to reduce the Eq.(1.1) to the equation with power non-linearity to use the known balancing principle. The balancing principle, determined in our previous work, works not only positive integer power but also negative and/or fractional powers. Moreover, the power is least numeral satisfying the finite expansion in most cases [16]. Hence in the similar manner, the novel balancing principle for non-power-law non-linearity is proposed

$$
N+n=q N+p s N,(\bmod n),
$$

where the highest order term is $\frac{\partial^{n} u}{\partial x^{n}}$ and the highest order nonlinear term is $\frac{\partial^{q} u}{\partial x^{q}}\left(\frac{\partial^{s} u}{\partial x^{s}}\right)^{p}$. By the novel proposed balancing principle (Eq. (1.2)), the power is obtained as the least numeral and positive integer, so the computational cost decreases. Additionally, the novel balancing principle works for all types of non-linearity and general cases. We exemplified some examples either does not work with already existing balancing principles in the literature. In this work, we consider the auxiliary equation method based on the Mathieu equation. In order to explain the idea of the auxiliary equation method, using the wave transformation,

$$
u(x, t)=u(\xi), \xi=x-\mu t .
$$

A nonlinear partial differential equation (PDE), $M\left(u, u_{x}, u_{t}, u_{x x}, u_{x t}, u_{t t}, \ldots\right)=0$, is reduced to a nonlinear ODE, $N\left(u, u_{\xi}, u_{\xi \xi}, \ldots\right)=0$. Assuming that the exact solution of equation nonlinear ODE has the simple finite expansion as

$$
u(\xi)=\sum_{i=0}^{N} g_{i} z(\xi)^{i},
$$

where $g_{i}$ are unknown constants to be determined later. Also, $z(\xi)$ defines the exact solution of the new proposed auxiliary equation. The unknown coefficients are determined in main three steps: (1) substituting the proposed auxiliary equation into the reduced nonlinear ODE. (2) Equating each coefficient of power of $z(\xi)$ to zero. (3) Solving the corresponding algebraic system, the coefficients are obtained. Also, the main step is determination of the integer $N$, which indicates the number of terms will be used in Eq.(1.4), basically by balancing the term with the highest order derivative and the term with the highest power non-linearity in reduced nonlinear ODE. But, in this work, the novel proposed balancing principle (Eq. (2)) is used to determine the integer so the considered generalized rod equation has non-power-law non-linearity. Generally, the function $z(\xi)$ is used as the exact solution of proposed auxiliary equation in the literature. Since nonlinear PDEs cannot be recovered by only one auxiliary ordinary differential equation, there have been many studies utilizing different exactly solvable auxiliary equations $[14,15]$.

As it is known that Eq. (1.1) has solitary and periodic type travelling wave equation, Mathieu equation [14],

$$
\frac{d^{2} z(\xi)}{d \xi^{2}}+(a-2 q \cos (\xi)) z(\xi)=0,
$$


which has a solution as $z(\xi)=C_{1}$ MathieuC $(a, q, \xi)+C_{2}$ MathieuS $(a, q, \xi)$, where MathieuC and MathieuS are periodic functions for countably many values of (a function of $q$ ). In this work, the rod equation is solved using the novel ansatz and the proposed method which has the same idea with different auxiliary equation.

\section{Solutions}

In this section, using proposed method and the novel balancing principle, the analytical solutions of the following generalized elastic rod equation is obtained

$$
u_{t t}-c_{0}^{2}\left(1+n a_{n}\left(u_{x}\right)^{2}\right)_{x x}-\frac{\nu^{2} J_{\rho}}{s} u_{t t x x}=F(t) .
$$

Till now, the solutions of the elastic rod equation (Eq. (1.1)) is obtained after reducing to ODE, transformation $u^{\prime}=v$ and integrations are used. Hence instead of the Eq. (1), the reduced equation is solved i.e. the original equation is not solved $[8,12,13,17,18,19]$. Using the novel balancing principle for non-power-law non-linearity, Eq. (1.1) is solved directly. To our knowledge, this study is the first attempt to investigate the analytical solutions of the generalized nonlinear elastic rod equation (Eq. (1.1)). With the wave transformation, $u(x, t)=u(\xi), \xi=x-\mu t$, Eq. (2.1) is reduced as below,

$$
\mu^{2} u^{\prime \prime}-c_{0}^{2} u^{\prime \prime}-n c_{0}^{2}\left(u^{\prime}\right)^{n-1} u^{\prime \prime}-\frac{\nu^{2} J_{\rho}}{s} \mu^{2} u^{(4)}=0,
$$

respect to the novel balancing principle (Eq. (1.2)) $N=\frac{4}{n}(\bmod 4)$.

Case 1.In the case $n=2$ as generally considered in the literature, using the novel balancing principle $N=\frac{4}{2}=$ $2(\bmod 4)$ is obtained, so the ansatz is $u(\xi)=g_{0}+g_{1} z(\xi)+g_{2} z(\xi)^{2}$. Applying the given procedure, the parameters are obtained as the solution of the nonlinear algebraic system.

$$
g_{1}=0, C_{1}=\frac{-C_{2} \text { MathieuSPrime }(a, q, \xi)}{\text { MathieuCPrime }(a, q, \xi)}, \mu=-c_{0} .
$$

The plot of the obtained solution is given by Figure 1. Figure 1. The 3D ((a)-(b)) and contour (c) plots of the solution of Eq. (2.2) via Mathiue approximation method for Case $1 g_{0}=2, g_{2}=1, s=2, c_{0}=\sqrt{3}, \nu=2, a=1, q=1.2, a_{n}=$ $1, J_{\rho}=1$.

Case 2.. As a novel case $n=4$ is considered, using the novel balancing principle $N=\frac{4}{4}=1(\bmod 4)$ is obtained, so the ansatz is $u(\xi)=g_{0}+g_{1} z(\xi)$. Applying the given procedure, the plot of the solution is given by Figure 2 .

Figure 2. The 3D ((a)-(b)) and contour (c) plots of the solution of Eq. (2.2) via Mathiue approximation method for Case $2 g_{0}=2, g_{1}=1, s=2, c_{0}=3, \nu=1, a=1.8, a_{n}=1, J_{\rho}=1, \mu=0.1$.

\section{Conclusion}

The main idea of this study is based on obtaining the exact solutions of generalized elastic rod equation containing non-power non-linearity by using the exact solutions of different type equations as an ansatz. By means of Mathieu equation with the wave transformation, the exact solutions of the generalized elastic rod equation are obtained. The generalized elastic rod equation is not an usual equation that contains parameters refer to physical and chemical properties of rod material. In this work, the novel balancing principle for non-power non-linearity to determine degree of ansatz is proposed for the first time in the literature. We believe that the obtained solutions of the generalized elastic rod equation will play key role in further analytical and numerical studies. Future studies will be based on investigating the analytical solutions of the nonlinear partial differential equations containing the non-power non-linearity via the proposed novel balancing principle in this study.

\section{Acknowledgment.}

We thank the reviewer for their insightful comments and suggestions that helped us improve the paper.

\section{Funding}

There is no funding for this work. 


\section{Availability of data and materials}

Not applicable.

\section{Competing interests}

The authors declare that they have no competing interests.

\section{Author's contributions}

All authors contributed equally to the writing of this paper. All authors read and approved the final manuscript.

\section{References}

[1] Abdou, M.A.: Exact travelling wave solutions in a nonlinear elastic rod equation. .Int. J. Nonlinear Sci. 7 (2), 167-173 (2009).

[2] Zhuang, W.,Guiltong, Y.: The propagation of solitary wave in a nonlinear elastic rod. Applied Mathematics and Mechanics . 7, 615-626 (1986).

[3] Zhuang, W., Zhang, S.Y.: The strain solitary wave in a nonlinear elastic rod. Acta Mech. Sin. 3 , 62-72 (1987).

[4] Duan,W.S., Zhao, J.B.: Solitary waves in a cuadratic nonlinear elastic rod. Chaos Solitons and Fract. 11, 1265-1267 (2000).

[5] Li, J., Zhang,Y.: Exact traveling wave solutions in a nonlinear elastic rod equation. Appl. Math. and Comp., 202, 504-510 (2008).

[6] Kabir,M.M.: Exact travelng wave solutions for nonlinear elastic rod equation. Journal of King Saud University-Science, 31 (3), 390-397 (2019).

[7] Gomez, C. A.,Garzon, H.G., Hernandez,J. C.:On Elastic Rod Equation with Forcing Term:Traveling Wave Solutions. Contemporary Engineering Sciences, 11 (4), 173 - 181 (2018).

[8] Dai, H.-H., Kowloon, Kong, H. :Model equations for nonlinear dispersive waves in a compressible Mooney-Rivlin rod. , Acta Mechanica 127, 193-207 (1998).

[9] $\mathrm{Li}$, L., $\mathrm{Hu}, \mathrm{Y} ., \mathrm{Li}, \mathrm{X}$.: Longitudinal vibration of size-dependent rods via nonlocal strain gradient theory. International Journal of Mechanical Sciences 115-116, 135-144 (2016).

[10] Zhuang,W., Zhang,G.T.: The propagation of solitary waves in a nonlinear elastic rod. Appl. Math. Mech. 7, 615-626 (1986).

[11] Lu, K.P., Guo,P., Zhang,L., Yi,J.Q., Duan,W.S.:Perturbation analysis for wave equation of nonlinear elastic rod. Appl. Math. Mech.27, 1233-1238 (2006).

[12] Dai,H.H., Huo,Y.: Solitary waves in an inhomogeneous rod composed of a general hyperelastic material. Acta Mech. 35, 55-69 (2002).

[13] Porubov, A.V., Velarde, M.G.:Strain kinks in an elastic rod embedded in a viscoelastic medium. Wave Motion 35 , 189-204 (2002).

[14] Pinar, Z., Kocak, H.:Exact solutions for the third-order dispersive-Fisher equations. Nonlinear Dynamics. 91 (1), 421-426(2018).

[15] Kocak, H., Pinar, Z.:On solutions of the fifth-order dispersive equations with porous medium type non-linearity. Waves in Random and Complex Media. 28 (3), 516-522 (2018).

[16] Pinar, Z., Öziş, T.:Observations on the class of "Balancing Principle" for nonlinear PDEs that can be treated by the auxiliary equation method. Nonlinear Analysis: Real World Applications. 23, 9-16(2015). 
[17] Baskonus, H. M., Bulut, H., Atangana, A. :On the Complex and Hyperbolic Structures of Longitudinal Wave Equation in a Magneto-Electro-Elastic Circular Rod. Smart Materials and Structures. 25(3), (2016).

[18] Baskonus, H. M., Gómez-Aguilar, J. F. :New Singular Soliton Solutions to the longitudinal Wave Equation in a Magneto Electro-elastic Circular Rod with Local M-derivative. Modern Physics Letters B. 33(21), 1-16(2019).

[19] Ilhan, O. A., Bulut, H., Sulaiman, T.A., Baskonus, H. M. :On the new wave behavior of the Magneto-Electro-Elastic (MEE) circular rod longitudinal wave equation. An International Journal of Optimization and Control: Theories and Applications 10(1), 1-8 (2020).

[20] Bulut, H., Sulaiman, T.A., Baskonus, H. M. :On the solitary wave solutions to the longitudinal wave equation in MEE circular rod. Opt. Quant. Electron. 50, (2018).

\section{Affiliations}

ZEHRA PINAR

AdDRESS: Tekirdağ Namık Kemal University, Dept. of Mathematics, 59030, Tekirdağ-Turkey.

E-MAIL: zpinar@nku.edu.tr

ORCID ID:0000-0002-9344-7308 